\title{
Globalización en crisis; por un desarrollo sostenible ${ }^{1}$ Globalization in crisis,; for a sustainable development
}

\author{
"Vivimos en una interconectividad como nunca hasta ahora \\ ha existido. Pero en este mundo hay cosas insoportables (...) \\ Se pueden ya identificar dos grandes retos: (por una parte) \\ la inmensa brecha que existe entre los muy pobres y los \\ muy ricos y que no cesa de crecer. Es una innovación de los \\ siglos XX Y XXI. Los muy pobres en el mundo de hoy ganan \\ apenas dos dólares por día. No podemos dejar que esa \\ brecha se amplíe aún más. Este sólo hecho debe generar \\ un compromiso; (por la otra) los derechos del hombre y el \\ estado del planeta (...).2
}

\section{Rolando Cordera Campos}

Coordinador del Programa Universitario de Estudios del Desarrollo. «cordera@unam.mx»
Journal of Economic Literature (JEL): O21, 054, 05

Palabras clave:

Economía internacional

Política de planificación

América Latina

Estudios económicos globales

Keywords:

International Economics

Planning Policy

Latin America

Economywide Country Studies

Fecha de recepción:

29 de abril de 2016

Fecha de aceptación:

27 de septiembre de 2016

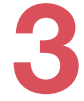

ECONOMÍAunam vol. 14 núm. 40, enero-abril, 2017

\section{Resumen}

Stéphane Hessel

Se advierte que el proceso de globalización vive una profunda crisis que pone en entredicho las supuestas ventajas que ofrecen sus paradigmas. La apertura comercial, el control de la inflación y del déficit público,así como el adelgazamiento de los estados y el predominio del mercado; solo han agudizado la pobreza y la concentración del ingreso. Por ello, se propone situar a la cuestión social en un lugar central, que se privilegie ala igualdad, al crecimiento económico y a la sostenibilidad ambiental, mediante una renovación y ampliación del Estado bajo un enfoque de derechos humanos; y así poder recuperar las dinámicas económicas, cuidar la cohesión social y evitar que el pluralismo democrático sea sofocado por el autoritarismo y las tendencias regresivas.

\section{Abstract}

The globalization process lives a deep crisis that calls into question the supposed advantages that offer their paradigms. Openness to trade, inflation and public deficit control, as well as thinning of the States and the market predominance have only exacerbated poverty and the income concentration. For this reason, it is proposed to position the social issues in a central place, emphasizing equality, economic growth and environmental sustainability through a renewal and expansion of the State under a human rights approach, so with this recover the economic dynamics, take care of social cohesion and avoid that democratic pluralism is stifled by authoritarian and regressive trends.

1 Una versión preliminar se presentó en el seminario "Los ODS en México y América Latina: retos comunes para una agenda compartida", 22 de abril de 2016.

2 Stéphane Hessel, "Indignez-vou", http://www.eldamoneo.com/indignate.pdf 
El proceso de globalización vive un punto de inflexión sin una trayectoria futura previsible. La crisis financiera estallada en 2008 con su interminable cauda de efectos sociales y políticos así como la amenaza cierta y actual del cambio climático, han puesto en la picota la supuesta ventaja de los paradigmas productivo y económico predominantes. La malhadada combinación de apertura externa sin control, baja inflación, déficit públicos por mandato, adelgazamiento de los Estados y predominio del mercado se ha traducido en más desigualdad, mayor pobreza en muchos países avanzados o en desarrollo y una aguda concentración del ingreso en unas pocas manos. ${ }^{3}$

El de la desigualdad es un desafío que vive y se reproduce en las entrañas del sistema económico moderno y le da actualidad a la idea del capitalismo como un mecanismo contradictorio e inestable que, dejado a su libre transcurrir, puede ser implacablemente autodestructivo y llevar al mundo en su conjunto a una circunstancia límite. Enfrentar a la desigualdad implica, de manera inexcusable, poner a la cuestión social en el centro de las ocupaciones nacionales y globales. La vieja cuestión social que indignara a Dickens no desaparece sino que se mueve a otras latitudes y la nueva, plagada de riesgos e incertidumbres, se implanta urbi et orbi sin que la primera desaparezca.

Enfrentar a la desigualdad implica, de manera inexcusable, poner a la cuestión social en el centro de las ocupaciones nacionales y

globales

Ahora parece claro que, sin contar con arquitecturas globales y nacionales que sean capaces de entender que para ser estrátegico, en el horizonte deben estar con claridad explícita la igualdad, el crecimiento económico y la sostenibilidad ambiental, el futuro aparece cuando menos esquivo y nublado. Por ello es que hay que celebrar los acuerdos de la Cumbre para el Desarrollo Sostenible, realizada en 2015.4 Se trata de esfuerzos que urge multiplicar y traducir en políticas de Estado porque los números rojos de nuestro rostro social son, lo siguen siendo, abrumadores.

Hablamos de políticas de Estado más que de políticas públicas porque el esfuerzo que hay que desplegar para lograr esos objetivos y metas tendría que ser a mediano y largo plazos a más de sistemático e integral. La recurrencia e insistencia en las políticas públicas suele llevar a soslayar la importancia del largo plazo y la necesidad de ver las intervenciones del Estado bajo una perspectiva y

Contar con arquitecturas globales y nacionales con claridad explícita la igualdad, el crecimiento económico y la sostenibilidad ambiental un enfoque integrales e integradores.

Asimismo, el uso indiscriminado de dicha noción de "políticas públicas" ha llevado a ignorar que la política-eje, central, es la política económica, convertida en la menos pública de dichas políticas.

La Comisión Económica para América Latina y el Caribe (CEPAL) dio a conocer su Panorama social de América Latina. Se trata de un informe completo y útil en relación con la cuestión social de nuestro continente. Entre

3 Datos del informe OXFAM, 2015, indican que la riqueza de los cuatro millonarios mexicanos más ricos representa $9 \%$ del PIB.

4 Cumbre para el Desarrollo Sostenible, realizada en septiembre de 2015, en la que se aprobaron 17 puntos, conocidos como objetivos de desarrollo sostenible, acuerdos que dan seguimiento a los Objetivos de Desarrollo del Milenio adoptados en 2000. 
otras cosas, Alicia Bárcena, secretaria ejecutiva de la Comisión, dijo que de 2010 a 2014 en la mayoría de los países había una disminución de la pobreza, con las excepciones de México y Costa Rica. El informe señala que en nuestro país la pobreza pasó de $51.6 \%$ en 2012 , a 53.2 en 2014 , mientras la pobreza extrema creció $0.6 \%$ y afecta a $20.6 \%$ del total la población. ${ }^{5}$

Ya el Consejo Nacional de Evaluación de la Política de Desarrollo Social (Coneval) nos había informado que en 2014, 55.3 millones de personas estaban en condición de pobreza (2.3 carencias sociales promedio); 6 de ellas 11.4 millones se encuentran en pobreza extrema (con 3.6 carencias sociales en promedio). Asimismo, que 8.5 millones de mexicanos viven por debajo de la línea de bienestar económico ("vulnerables por ingreso"); hay 31.5 millones de personas con 1.8 carencias promedio (vulnerables por carencia social), y únicamente $20.5 \%$ de la población nacional (24.6 millones), no es pobre ni vulnerable por ingreso o carencia social.

\begin{tabular}{|r|}
\hline Figura 1 \\
2014: pobreza \\
multidimensional \\
\hline
\end{tabular}

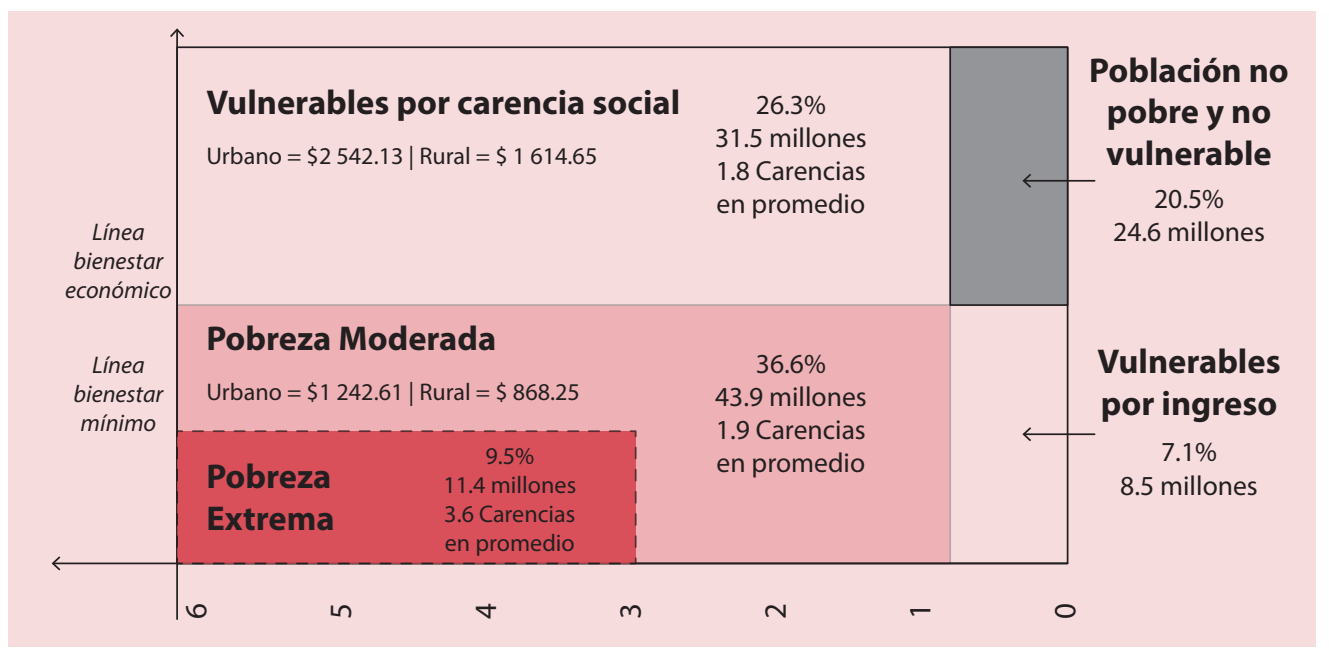

Fuente: estimaciones del Coneval con base en el MCS-ENIGH 2014.

México devino en una sociedad de ingresos

bajos generalizados y una concentración de los ingresos

y la riqueza, por lo menos

similar a la del pasado previo al cambio estructural globalizador
Éstos y otros números similares sugieren que, sin haber sido nunca una sociedad afluente, México devino en una sociedad de ingresos bajos generalizados y una concentración de los ingresos y la riqueza, por lo menos similar a la del pasado previo al cambio estructural globalizador. El gran desafío que tenemos sigue siendo traducir en estrategias y políticas justicieras y consistentes los reclamos sociales y el conocimiento acumulado sobre la morfología y dinámica de la cuestión social contemporánea. Las respuestas no son sencillas;

5 No hay que perder de vista que la reducción de la pobreza registrada encara hoy los embates de la recesión en el Cono Sur y sus implicaciones no auguran nada bueno.

6 El Consejo Nacional de Evaluación de la Política Social (Coneval) considera seis indicadores sociales para sus mediciones de vulnerabilidad de las personas en pobreza, éstos son: rezago educativo; acceso a los servicios de salud; acceso a la seguridad social; calidad y espacios de la vivienda; acceso a los servicios básicos en la vivienda y acceso a la alimentación. 
empero, para nuestro caso el contenido del artículo primero constitucional traza una ruta clara en línea con los Objetivos de Desarrollo Sostenible (ODS) cuyas metas tienen que ver de principio a fin con el desarrollo.

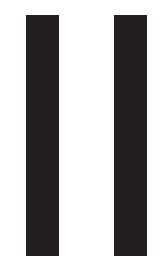

Estamos frente a la necesidad de definir una nueva agenda global de desarrollo en su acepción más extensa, es decir, una agenda que contemple los nuevos desafíos políticos, económicos, sociales y ambientales y las prioridades (...) se requiere de una fortalecida institucionalidad financiera global para enfrentar la crisis; y una arquitectura institucional legítima y representativa, cimentada en un multilateralismo real y efectivo, que sea capaz de dar cuenta del cumplimiento de las decisiones globales. ${ }^{7}$

En nuestro caso, asumir los objetivos (poner al país en ruta con lo firmado) debería enfatizar: revisar metas y programas gubernamentales. Podríamos, realistamente, plantear aumentar coberturas en salud y educación; también, reducir el número de pobres mediante una estrategia de inversión productiva pública, activar el mercado interno y generar aceleradamente el número de empleos buenos y dignos, cuya ausencia afecta en especial a los jóvenes y los adultos jóvenes, quienes al no encontrar cobijo en el mercado de trabajo, optan por la informalidad, la emigración o -la peor de las fugas-, a la informalidad criminal organizada.

No está de más tener presente que en los últimos años la generación de trabajos remunerados con más de tres salarios mínimos ha declinado mientras que los empleos remunerados con hasta tres salarios mínimos han aumentado. Esta falta de empleo bueno debe inscribirse en el panorama demográfico del país; de acuerdo con el Consejo Nacional de Población (Conapo), 65.1\% de la población nacional (en 2012) tenía entre 15 y 64 años, mientras que los jóvenes de 12 a 29 años representaban $32.1 \%$; se prevé que para el año 2050 la relación entre la población adulta mayor y la población joven será prácticamente de uno a uno, lo que implica que el llamado bono demográfico, de seguir las cosas como van, puede convertirse en un pagaré que el país no podrá solventar.

Al mismo tiempo, la llamada "brecha laboral" se ha tornado falla geológica. Con base en una metodología elaborada en $2015^{8}$ es posible ver los requerimientos reales de nuevos empleos; como se aprecia en la siguiente figura, elaborada por investigadores del Centro de Estudios Económicos del Sector Privado (CEESP), la fuerza laboral potencial en México es de más de 58 millones, la cual resulta de sumar la PEA con la población no económicamente activa disponible. Al sumar el número de personas que se encuentran en desocupación, las que están en subocupación ${ }^{9}$ y los no ocupados disponibles (tienen interés de trabajar, pero han dejado de buscar porque piensan

7 Alicia Bárcena, "La gobernanza global para enfrentar un cambio de época”, septiembre 2011, disponible en http://www.embajadaabierta.com/?p=309

8 David Blanchflower y Andrew Levin, "Labor Market Slack and Monetary Policy”, en http://www. nber.org/papers/w21094

9 INEGI considera como subocupados a las "personas de 15 y más años de edad que tienen la necesidad y disponibilidad de ofertar más horas de trabajo de lo que su ocupación actual les permite". 
que ya no tienen oportunidad), resulta que tenemos un déficit de empleos mayor a $20 \%$ respecto a la fuerza laboral potencial.

Figura 2

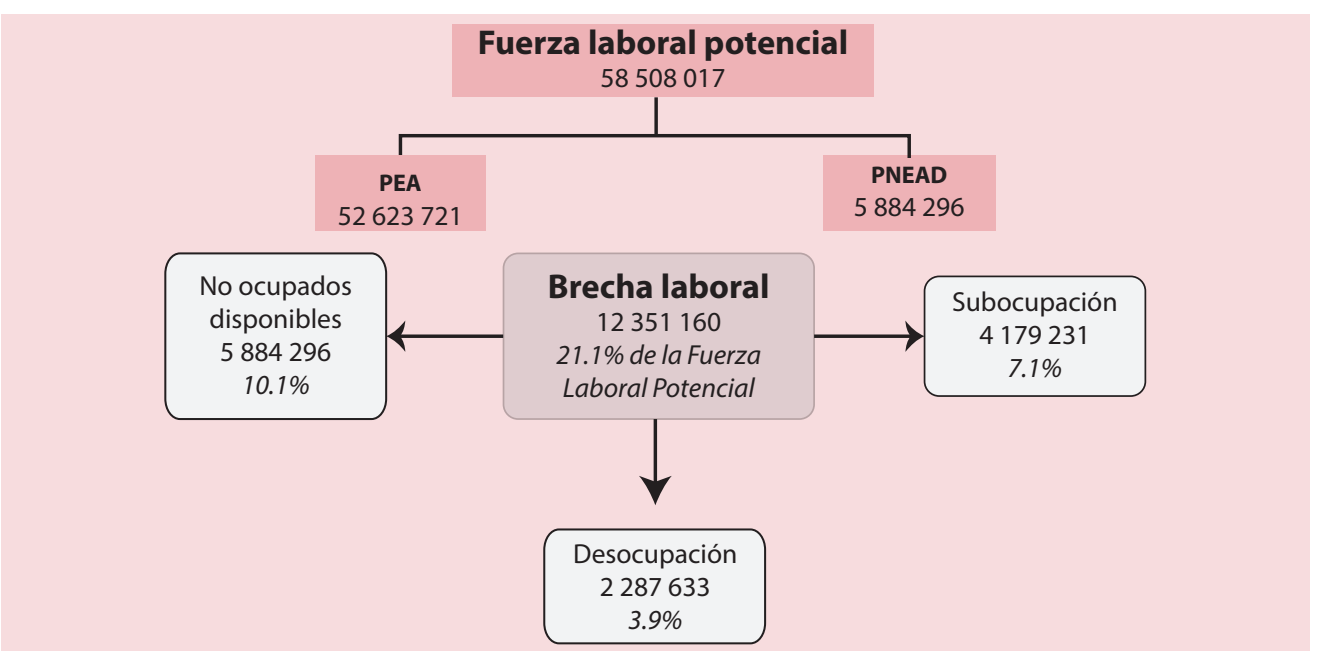

Fuente: CEESP, 2015.

Figura 3

México: Brecha laboral, 2005.I-2015.IV

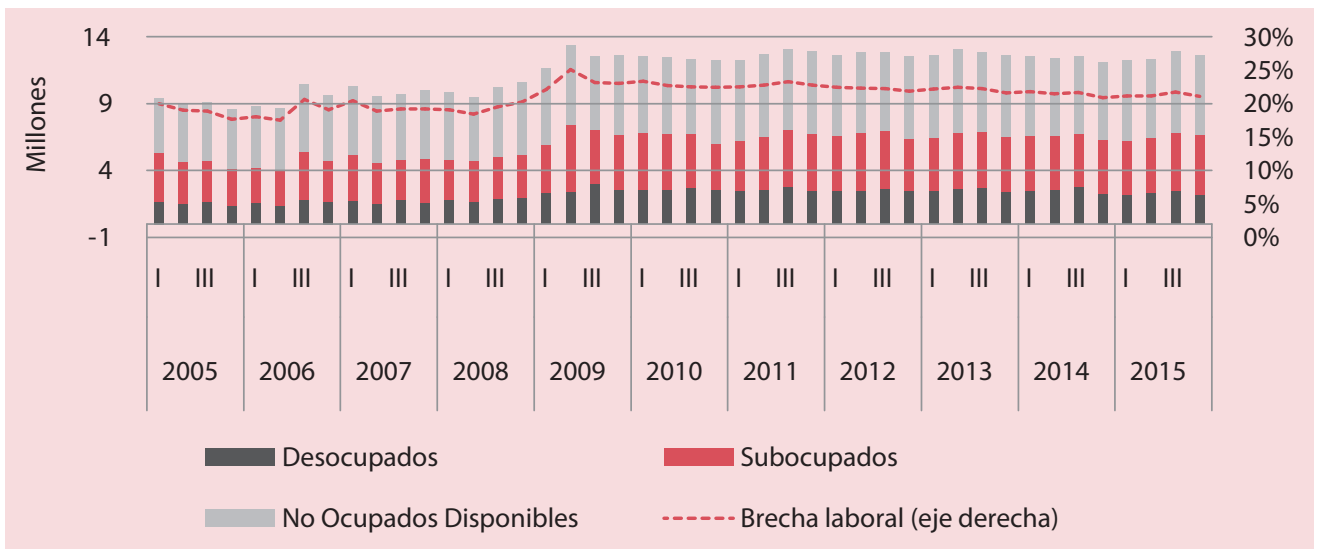

Fuente: elaboración con base en ENOE (2016) y metodología del CEESP (2015).

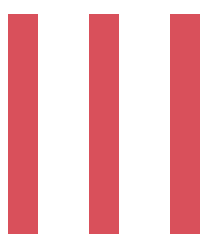

En esta perspectiva, asumir la necesidad de construir un nuevo curso de desarrollo es una aspiración genuina que no tiene que ver con "buenos deseos". Guarda una relación directa con el cumplimiento de los ODS y nos exige plantearnos una vuelta al Estado conceptual, política e institucionalmente hablando. Es conveniente recordar que el gran compromiso histórico del capitalismo de la segunda posguerra resumido en el vocablo "Estado de bienestar", probó su eficacia histórica. No debería haber mayor discusión conceptual sobre esto, sin menoscabo de las muchas cuestiones específicas que la propia evolución de la sociedad y su demografía le han planteado al esquema original del Estado protector. 
El gran compromiso histórico

del capitalismo de la segunda posguerra resumido en el vocablo "Estado de bienestar", probó su eficacia histórica
Sin embargo, la fase actual de incertidumbre que ha sucedido a la Gran Recesión, no sólo amenaza al mundo con un regreso del declive económico o la implantación sin más de una pauta de "estancamiento secular" sino también con la agudización de los embates contra el Estado social. Sostén básico de la demanda efectiva y soporte de los arreglos sociales destinados a modular el conflicto y darle estabilidad política y social a un sistema económico proclive a gestar grandes y pequeños desequilibrio. El Estado social es imprescindible hoy, para recuperar las dinámicas económicas, cuidar la cohesión social y evitar que el pluralismo democrático sea sofocado por el autoritarismo y las tendencias regresivas en la observación y respeto a los derechos humanos.

En México, sin haber avanzado significativamente en la cons-

El Estado social es trucción de una forma estatal de esa especie, cuyos compromisos imprescindible hoy, para recuperar las dinámicas económicas, cuidar la cohesión social y evitar que el pluralismo democrático sea sofocado por el autoritarismo y las tendencias regresivas sociales fueran parte explícita de la arquitectura democrática y constitucional del Estado, se recurre a la menor provocación al espectro de la inestabilidad fiscal y financiera para insistir en revisiones drásticas en materia de derechos sociales y laborales.

En este sentido, quizá resulte conveniente pensar el regreso del Estado no como una vuelta sino como la consecuencia de una dialéctica que no ha logrado su síntesis: frente al „demasiado Estado", el reclamo libertario de afirmación de la individualidad y de la autonomía; frente a una excesiva condensación de la política en partidos y parlamentos, las nuevas figuras políticas e ideológicas, culturales en un sentido amplio, emanadas de la globalización y del fin de la bipolaridad: sociedad civil, derechos humanos como mandato universal, cosmopolitismo democrático, ciudadanía global.

El retorno del Estado así, tiene que darse mediante su reforma No menos Estado sino su y la expansión de la política democrática. Ciertamente, esta recurenovación y ampliación, peración seguirá sujeta a las limitaciones emanadas de las historias que es lo que la sociedad mundiales y nacionales, así como de las experiencias específicas, globalizada reclama idiosincráticas, de las formaciones nacionales. Pero, en lo general, puede proponerse esta fórmula: no menos Estado sino su renovación y ampliación, que es lo que la sociedad globalizada reclama. No menos sector público sino más, sostenido ahora en formas efectivas de descentralización administrativa y deliberación política, es lo que se impone si la globalización alcanzada en los últimos lustros va a dar paso a un proceso fructífero y bajo control de los estados y las sociedades.

Un Estado de bienestar y no un Estado subsidiario, que avance hacia una estructura tributaria y un sistema de transferencias que privilegie la solidaridad social. Con una nueva ecuación Estado-mercado-sociedad se podrá alcanzar un desarrollo con empleos de calidad, cohesión social y sostenibilidad ambiental:10

10 Comisión Económica para América Latina y el Caribe (CEPAL), "La hora de la igualdad: brechas por cerrar, caminos por abrir", 2010, p. 11. 
Así resume la CEPAL lo que podría ser el inicio de una nueva síntesis de la economía política del desarrollo. La dialéctica referida no apunta sino a la parálisis, el estancamiento, la anomia. De aquí la urgencia de encontrar, de imaginar al Estado necesario.

La reforma del Estado que la época reclama para encaminarse La reforma del Estado que a un cambio fundamental progresivo debe tener como eje maestro la época reclama para una reforma social del propio Estado. No puede reducirse a satisfaencaminarse a un cambio fundamental progresivo debe

tener como eje maestro una reforma social del propio Estado cer demandas específicas de cambios en el uso de los recursos o la conformación institucional; para ser un componente y un catalizador de una efectiva y radical "reforma de las reformas", debe centrarse en la reconstrucción de los tejidos y procesos sociales básicos que implica, a su vez, una redistribución del poder, un reacomodo radical de las relaciones y pesos entre las esferas de la economía y su comando en la asignación de los recursos y la distribución de los ingresos y la riqueza.

La igualdad de derechos provee el marco normativo y sirve de fundamento a los pactos
sociales (...) La agenda de igualdad exige (...) generar una institucionalidad que permita
consolidar un orden democrático y participativo (...) Dicha agenda pasa por construir un
gran acuerdo económico-social cuya expresión última es el pacto fiscal (...) implica una
estructura y una carga tributarias que permitan fortalecer el rol redistributivo del Estado y
de la política pública a fin de garantizar umbrales determinados de bienestar (...). ${ }^{11}$

Con el propósito de dar orden y sentido al gasto público, México en particular, requiere una gran reforma hacendaria-fiscal como necesario sustento de un pacto social abiertamente redistributivo a la vez que desarrollista. Para poder justificar socialmente el incremento en la recaudación, darle viabilidad política, se deben hacer explícitos los fines -tanto sociales como productivos- y asumir compromisos claros sobre la utilización de los recursos y con la rendición de cuentas. Así, los criterios más rigurosos de evaluación de la estrategia de desarrollo y de la política económica y social, serán la equidad, la remoción sostenida de la pobreza y los avances efectivos hacia la igualdad, en un contexto de innovación y expansión productiva.

En razón de ello es que asumir los ODS constituye el punto de partida para el diseño de una estrategia destinada a responder a un objetivo central cuyo cumplimiento debe empezar a materializarse de inmediato: el establecimiento de un sistema de bienestar basado en derechos económicos y sociales exigibles, de alcance universal, y especialmente orientado a la promoción de la protección y el bienestar juvenil, sin descuidar la atención a otros grupos vulnerables de la sociedad, también directamente afectados por la crisis y sus secuelas. Este propósito debe guiar la construcción de una nueva forma de desarrollo que se despliegue en un crecimiento económico alto y sustentable, así como en una progresiva redistribución social y regional del ingreso.

No es ajeno el hecho de que México registra un alto nivel de incumplimiento de sus compromisos internacionales en materia de derechos humanos, a todo lo largo de su definición, pero en especial en lo tocante a los derechos económicos y

11 CEPAL, "La hora de la igualdad...", cit., p. 257. 
sociales. Tampoco nos es ajeno que el país sufre un alto grado de desprotección de sus habitantes, tanto en materia de seguridad personal y pública como en materia social, donde se recogen déficit impresentables: tanto en el acceso garantizado a las pensiones y la jubilación, como a la salud e incluso a la alimentación y la nutrición necesarias y adecuadas.

Por ello hay que insistir en que la ruta para una economía y un

Entender y asumir que la desarrollo diferentes sólo tiene una vía; en primer término, entender construcción de regímenes de y asumir que la construcción de regímenes de bienestar y protección bienestar y protección social, social, bajo un enfoque de derechos humanos, es uno de los temas bajo un enfoque de derechos centrales de las agendas democráticas, pero también de las agenhumanos, es uno de los temas das económicas para un desarrollo entendido como un proceso de centrales de las agendas cambio social y pedagogía democrática. Una empresa de este corte, democráticas debe verse como una condición sine qua non para darle materialidad y credibilidad a la idea de hacer de ésta la "hora de la igualdad", 12 y ahora puesta en sintonía con el logro de los objetivos de los ODS.

Como sociedad global requerimos ser capaces de inscribir la disminución de la desigualdad, el cuidado del medio ambiente y la superación de la poHabría que hablar de una breza como el meollo de la economía política internacional. De no hacultura global de la riqueza, de cerlo más que de una cultura de la pobreza, de la que nos hablaron un regodeo con la satisfacción los antropólogos en los años sesenta del siglo XX, habría que hablar de que se impone a desarrollados una cultura global de la riqueza, de un regodeo con la satisfacción que y no desarrollados se impone a desarrollados y no desarrollados.

Esta disonancia habla del gran divorcio y la insensibilidad que abruman y pueden ahogar a las sociedades en una "normalidad" que lleva a lo peor de los mundos:

(...) la idea de ciudadanía, dice Victoria Camps, no debe ir ligada exclusivamente a unos derechos individuales, sino que debe incluir al mismo tiempo aquellos vínculos capaces de unir a los ciudadanos con la comunidad. Esos vínculos, de los que había hablado en especial Rosseau, comprometerían al ciudadano con una serie de obligaciones, deberes cívicos, y constituirían lo que podríamos llamar 'la dimensión moral de la democracia'.13

Ya debemos dejar de seguir "entretenidos" en deplorar situaciones que consentimos:

(...) el sentimiento extendido de que las desigualdades son 'excesivamente profundas' y hasta 'escandalosas', coexiste con una aceptación implícita de sus múltiples expresiones específicas, así como con una sorda resistencia a corregirlas en la práctica. De ahí que el descontento social susceptible de ser mayoriario puede ir unido a una pasividad

12 CEPAL, "La hora de ..." op. cit.

13 Txetxu Aguado, La tarea política. Narrativa y ética en la España posmoderna, p. 86 en https:// books.google.com.mx/books?id=brDBWOXKslsC\&pg=PA82\&lpg=PA82\&dq=pacto+social+vi ctoria+camps\&source=bl\&ots=ILoGZyelTI\&sig=i6e1fMZjy1JmwPTpw1nfH_2rUkU\&hl=es\&sa= X\&ved=0ahUKEwjV34GP0qzMAhVIsIMKHWUfDLYQ6AEIGjAA\#v=onepage\&q=pacto\%20social\%20victoria\%20camps\&f=false 
práctica frente al sistema general de las desigualdades (...) De modo que existe un rechazo global de una forma de sociedad que coexiste con una forma de aceptación de los mecanismos que la reproducen. Propongo llamar paradoja de Bossuet a esta situación en la que los hombres en general se lamentan de aquello que aceptan en particular. ${ }^{14}$

Sólo mediante los acuerdos amplios y generosos de cooperación entre fuerzas sociales, grupos productivos y regiones, capaces de combinar la democracia con la movilización colectiva y la deliberación, será posible redefinir los linderos entre lo público y lo privado.

Justamente en las antípodas del individualismo posesivo es de ley reconocer, afirma
la filósfa española Adela Cortina, 15 que nos constituye nuestro ser con otros (...) si el
esfuerzo personal es imprescindible, también somos lo que somos por nuestra relación
con otros. Y, en un universo global, esto vale no sólo en cada comunidad política, sino
en el contexto de la globalidad: es de justicia distribuir globalmente los bienes que las
sociedades pueden dispensar (alimento, agua, atención sanitaria...) porque son sociales.

La clave reside en montar Tanto la desprotección como la falta de garantías de los derechos una conversación virtuosa entre acumulación de capital fundamentales y las enorme brechas sociales que se resumen en la y distribución de los frutos del cohabitación de la pobreza masiva y la aguda, inconmovible, desigualdad económica y social, deberían ser argumentos definitivos, prima facie diría algún jurista, para llevar a cabo una profunda transformación del Estado dirigida a la construcción de un régimen en condiciones de cumplir con el mandato contitucional de garantizar derechos fundamentales exigibles. Ésta debería ser la base jurídica y política para la construcción de un nuevo curso de desarrollo capaz de conjugar un crecimiento económico sostenido con transformaciones sociales de calado diverso pero dirigidas todas ellas a concretar un horizonte de igualdad social efectivo y durable. La clave reside -entonces- en montar una conversación virtuosa entre acumulación de capital y distribución de los frutos del crecimiento. Bajo el signo y el cemento de un entendimiento claro y a fondo de la cuestión social contemporánea, es que podremos volver a (re)conocer al desarrollo como un derecho central y fundamental de una modernidad globalizada en clave democrática.

14 Pierre Rosanvallon, La sociedad de los iguales, España, RBA Libros, 2012, pp. 15, 16 y 367. “Dios se ríe -afirmaba el magistrado y religioso francés- de los hombres que se quejan de las consecuencias, y en cambio consienten sus causas" paradoja que, dice el politólogo francés, "puede entenderse como una falacia de composición: la insatisfacción puede ser la suma de apreciaciones heterogéneas, mientras que los objetos o las razones de actuar deben estar positivamente determinadas".

15 Adela Cortina, "Ética pública desde una perspectiva dialógica" en https://repositorio.uc.cl/bitstream/handle/11534/6502/000507980.pdf?sequence=1\&isAllowed=y 


\section{Bibliografía}

BÁRCENA, Alicia, "La gobernanza global para enfrentar un cambio de época", septiembre 2011, disponible en http://www.embajadaabierta.com/?p=309

BLANCHFLOWER, David y Andrew LeVIN, Labor Market Slack and Monetary Policy, en http://www.nber.org/ papers/w21094

Centro de Estudios Económicos del Sector Privado (Ceesp), Metodología, 2015.

COMISIÓN ECONÓMICA PARA AMÉRICA LATINA Y EL CARIBE (CEPAL), La hora de la igualdad: brechas por cerrar, caminos por abrir, 2010, p. 11.

Consejo nacional de Evaluación de la Política Social (Coneval), el Módulo de Condiciones Socioeconómicas y la Encuesta Nacional de Ingresos y Gastos de los Hogares (MCS-ENIGH) 2014.

CORTINA, Adela, "Ética pública desde una perspectiva dialógica" en HESSEL, Stéphane, Indignez-vou, http://www.eldamoneo.com/indignate.pdf https://books.google.com.mx/books?id=brDBWOXKslsC \&pg=PA82\&lpg=PA82\&dq=pacto+social+victoria+camps\&source=bl\&ots=ILoGZyelTI\&sig=i6e $1 \mathrm{fMZj}$ y1JmwPTpw1nfH_2rUkU\&hl=es\&sa=X\&ved=0ahUKEwjV34GP0qzMAhVIsIMKHWUfDLYQ6AEIGjAA $\# \mathrm{v}=$ onepage \&q=pacto\%20social\%20victoria\%20camps\&f=false https://repositorio.uc.cl/bitstream/ handle/11534/6502/000507980.pdf?sequence=1\&isAllowed=y

HESSEL, Stéphane, Indignez-vou, http://www.eldamoneo.com/indignate.pdf INEGI, La Encuesta Nacional de Ocupación y Empleo, ENOE, 2016.

ORgANIZACIÓN DE LAS NACIONES UNIDAS (ONU), Objetivos de Desarrollo del Milenio, 2000.

OXFAM, Informe temático, Tenerlo todo y querer más, 2015, Oxfam House, Cowley, Oxford, OX4 2JY, UK. Pierre Rosanvallon, La sociedad de los iguales, España, RBA Libros, 2012, pp. 15, 16 y 367.

Txetxu Aguado, La tarea política. Narrativa y ética en la España posmoderna, p. 86 en https://books. google.com.mx/books?id=brDBWOXKslsC\&pg=PA82\&lpg=PA82\&dq=pacto+social+victoria+ camps\&source=bl\&ots=ILoGZyelTI\&sig=i6e1fMZjy1JmwPTpw1nfH_2rUkU\&hl=es\&sa=X\&ved =0ahUKEwjV34GP0qzMAhVIsIMKHWUfDLYQ6AEIGjAA\#v=onepage\&q=pacto\%20social\%20 victoria $\% 20$ camps\&f=false 